\title{
Ampicillin-Chloramphenicol-Resistant Haemophilus influenzae: Plasmid-Mediated Resistance in Bacterial Meningitis
}

\author{
GARY D. OVERTURF, DOUGLAS CABLE, AND JOEL WARD \\ Department of Pediatrics, University of California, Los Angeles School of Medicine, Los Angeles, California \\ 90024; Olive View Medical Center, Sylmar, California 91342 [G.D.O., D.C.]; and \\ Harbor-UCLA Medical Center, Torrance, California 90509 [J.W.]
}

\begin{abstract}
A 4-month-old infant with congenital heart disease and sepsis and arthritis, and subsequently meningitis, caused by an antibiotic-resistant strain of Haemophilus influenzae type $b$, failed to respond to sequential therapy with ampicillin and trimethoprim/sulfamethoxazole. Following treatment with ceftizoxime, the infant was well for 42 days, until he returned to the hospital and died. A total of 10 Haemophilus influenzae type b isolates, all outer membrane protein subtype $5 \mathrm{~L}$, was isolated from the pretreatment blood and synovium, cerebrospinal fluid and subdural fluids, and the petrous pyramids at autopsy. Pretreatment isolates had no detectable plasmid DNA, chloramphenicol acetyltransferase or $\beta$-lactamase; the minimal inhibitory concentration for ampicillin (AM) and chloramphenicol (CM) was 0.2 and $0.8 \mu \mathrm{g} / \mathrm{ml}$, respectively. However, all cerebrospinal fluid isolates had a 42-44 $\mathrm{mD}$ plasmid and produced chloramphenicol acetyltransferase and $\beta$-lactamase; the minimal inhibitory concentration of these isolates to $A M$ and $C M$ were 12.5 and $25 \mu \mathrm{g} / \mathrm{ml}$, respectively, and were also resistant to tetracycline and sulfonamide. Resistance to $A M$ and $C M$ was cotransferred by filter-mating conjugation at a frequency of one to two transconjugants per $10^{5}$ to an Rd haemophilus recipient. Posttreatment isolates from the petrous pyramids also were resistant to $\mathrm{AM}$ and $\mathrm{CM}$ and produced chloramphenicol acetyltransferase and $\beta$-lactamase activity, but had no plasmid DNA. These findings and data from genetic studies suggested that plasmid-bearing antibiotic-resistant $\mathrm{Hae}$ mophilus influenzae type b was selected from a heterogenous population, and that the $\mathrm{AM} / \mathrm{CM}$ resistance transposons were incorporated into the bacterial chromosome. (Pediatr Res 22: 438-441, 1987)
\end{abstract}

\section{Abbreviations}

CAT, chloramphenicol acetyltransferase

BL, $\beta$-lactamase

MBC, minimal bactericidal concentration

mD, megadaltons

AM, ampicillin

CM, chloramphenicol

Hib, Haemophilus influenzae, type b

OMP, outer membrane protein

SMX, sulfamethoxazole

TMP, trimethoprim

CSF, cerebrospinal fluid

MIC, minimal inhibitory concentration

Received December 3, 1986; accepted May 13, 1987.

Correspondence Gary D. Overturf, M.D., Olive View Medical Center, 14445 Olive View Drive, Rm. 3A108, SyImar, CA 91342.
WBC, white blood cell count

BHI, brain heart infusion

SDS, sodium dedecyl sulfate

Prior to 1974 , strains of Hib were consistently susceptible to AM (1). However, in 1974 Tomeh et al. (2) described three children with disease due to AM resistant strains. The mechanism for AM resistance in most strains of encapsulated and nonencapsulated strains of haemophilus has been the production of a TEM type $\mathrm{BL}$ encoded by conjugative plasmids (3-5). Currently, 20 to $30 \%$ of Hib strains and as many as 30 to $50 \%$ of unencapsulated strains produce $\mathrm{BL}(5)$. Plasmid-mediated resistance has also been demonstrated for CM-resistant populations of haemophilus (6), usually due to the production of a CAT encoded on conjugative plasmids. CM-resistant strains are frequently multiply resistant and cotransfer resistance to other antibiotics such as tetracycline, sulfonamides, and AM (3). In the following report, an infant with complicated disease due to Hib failed successive courses of therapy with AM and TMP/SMX and finally was cured with administration of ceftizoxime; the causative organism was a multiply antibiotic-resistant strain which caused relapsing episodes of invasive disease and respiratory colonization (i.e. middle ear) over a 4-month period.

\section{CASE REPORT}

On April 1, 1982 a 4-month-old male infant initially was admitted to a Los Angeles hospital with swelling of the right wrist on April 1, 1982. His past medical history included the diagnosis of congenital transposition of the great vessels, partially corrected by balloon atrial septostomy on the 1st day of life. He had never exhibited signs or symptoms of congestive heart failure or endocarditis. The child was treated with intravenous AM for a period of 14 days for septic arthritis. Hib was isolated from an aspiration of the wrist and blood culture. The clinical laboratories reported that these initial isolates were sensitive to AM and $\mathrm{BL}$ negative. The infant was discharged without further antibiotic therapy; he did not receive rifampin prophylaxis.

Four days after completing therapy the child was readmitted to the hospital with complaints of fever, lethargy, irritability, and poor feeding. Cerebrospinal fluid obtained at this time had 1116 $\mathrm{WBC} / \mathrm{mm}^{3}, 58 \%$ neutrophils, a glucose of $53 \mathrm{mg} / \mathrm{dl}$, a protein of $33 \mathrm{mg} / \mathrm{dl}$, and gram-negative bacillary bacteria. Therapy was initiated with CM and was continued when a CSF isolate of Hib was reported to be BL positive; routine CM susceptibility studies were not performed at that time. His mental status and clinical signs improved and a lumbar puncture obtained following the 
first day of CM therapy revealed 559 white blood cells, $73 \%$ neutrophils, and a gram-negative stain. However, by the 3rd hospital day, this lumbar puncture was reported to be growing Haemophilus influenzae. Determination of the MIC against chloramphenicol in the clinical laboratory was $16 \mu \mathrm{g} / \mathrm{ml}$. A lumbar puncture performed on the third hospital day revealed 206 white blood cells, 54\% neutrophils, and a gram-negative stain; however, the subsequent culture was positive again on the following day and therapy was initiated with intravenous TMP/ SMX $(20 \mathrm{mg} / \mathrm{kg} /$ day as TMP). Twenty-four $\mathrm{h}$ after initiation of TMP/SMX, his spinal fluid was sterile and remained sterile until the 5th day of TMP/SMX (i.e. 9th hospital day). During this time the child's mental status improved and meningeal signs abated, although persistent daily fever spikes to as high as $38.6^{\circ}$ $\mathrm{C}$ continued to occur and an increasingly full anterior fontanelle was appreciated. A lumbar puncture on day 10 of TMP/SMX revealed $266 \mathrm{WBCs}$, with $30 \%$ neutrophils, protein $37 \mathrm{mg} / \mathrm{dl}$, and glucose $32 \mathrm{mg} / \mathrm{dl}$. Although the the gram stain was negative, the culture subsequently grew Hib. At this time ceftizoxime (200 $\mathrm{mg} / \mathrm{kg} /$ day) was begun and a computerized tomographic scan was obtained, revealing a large subdural effusion with minimal midline shift. A subdural tap returned cloudy fluid with 11,900 $\mathrm{WBC} / \mathrm{mm}^{3}, 98 \%$ neutrophils, and a positive gram stain and culture for $H$. influenzae. Subdural taps were continued for 5 days at which time a drain was placed. Within $48 \mathrm{~h}$ of initiation of ceftizoxime all cultures of subdural taps and lumbar punctures were sterile and the drain was removed $72 \mathrm{~h}$ after placement. The infant defervesced on ceftizoxime and the therapy was continued for a total of 14 days and resolution of all clinical signs and symptoms occurred. No other significant site of persistent infection was identified. Rifampin prophylaxis was not prescribed.

Six weeks after discharge the child returned to the hospital and was admitted with a radiographically appreciated bilateral interstitial pneumonia. AM was administered and the child responded satisfactorily. Sputum cultures grew Staphylococcus aureus and a BL positive CM-resistant Hib.

Four wk later, during a routine cardiology clinic appointment, the child arrived with fever and was found to be hypotensive at the time of examination by the attending physician. CSF obtained during resuscitative efforts had $20 \mathrm{WBC} / \mathrm{mm}^{3}$ with two neutrophils. Subsequent cultures of blood and CSF were sterile. The peripheral white blood count was $24,300 / \mathrm{mm}^{3}$ with $31 \%$ segs, $5 \%$ bands, and 5,000 platelets $/ \mathrm{mm}^{3}$. Within $8 \mathrm{~h}$ after admission to the hospital the child died. Autopsy revealed no endocarditis or other site of chronic abscess or persistent infection. Examination of the spleen, thymus, and lymph nodes were normal. However, purulent material was identified bilaterally in the middle ears at the time of resection of the temporal pyramids; bilateral cultures grew Hib. Cultures taken at autopsy of the right wrist, meninges, and CSF were sterile.

\section{METHODS}

Antimicrobial susceptibility. Disk susceptibility testing was performed on Mueller Hinton-5\% horseblood chocolate agar plates supplemented with $1 \%$ isovitalex (7). Susceptible zones were defined in accord with NCCLS standards. MIC and MBC were performed in macrotube dilution tests in modified Levinthal broth (8). Disc susceptibility studies against TMP/SMX were performed according to the methods described by the manufacturer (9) utilizing 5\% horse blood in trytophan-depleted Mueller-Hinton media.

Typing and OMP determination. All isolates were type $\mathrm{b}$ as determined by standard agglutination procedures, with specific rabbit antisera. The outer membrane protein subtyping was performed by the method of Loeb et al. (10). Reference strains were kindly provided by Drs. Dan Granoff and Stephen Barenkamp, Washington University, St. Louis, MO (11).

$C A T$ and $B L$ activity. BL activity of each isolate was deter- mined by the rapid acidometric method of Thornsberry and Kirven (12). CAT activity was determined by the method described by Burns et al. (13).

Identification of plasmids and DNA electrophoresis. Screening for plasmid DNA was performed by a modification of the method of Meyers et al. (14). Briefly, haemophilus strains were grown overnight in $30 \mathrm{ml}$ of NAD, hemin and histidine-supplemented $\mathrm{BHI}$ broth and harvested by centrifugation. Pellets were resuspended in $1.5 \mathrm{ml} 25 \%$ sucrose $/ 50 \mathrm{mM}$ tris $\mathrm{Cl} / 1 \mathrm{mM}$ EDTA $(\mathrm{pH}$ 8.0 ) and sequentially exposed to $0.2 \mathrm{ml}$ of lysozyme $(10 \mathrm{mg} / \mathrm{per}$ $\mathrm{ml}), 100 \mu \mathrm{l} 250 \mathrm{mM}$ EDTA, $100 \mu \mathrm{l} 20 \%$ SDS, and $1 \mathrm{M} \mathrm{NaCl}$. Lysed preparations were incubated $\left(4^{\circ} \mathrm{C}\right)$ overnight and centrifuged; supernatants were treated with RNAse. Plasmid and chromosomal DNA was extracted by sequential phenol extractions, followed by cold ethanol precipitation $\left(-20^{\circ} \mathrm{C}\right)$. DNA electrophoresis was performed in 0.7 agarose gels and the molecular weight of visualized plasmids were estimated by comparison to concurrently electrophoresed Escherichia coli plasmid standards.

Conjugation and transformation. Log phase donor and recipient cells ( $10^{8}$ cells each) were mated on filters (Millipore, GS, $0.22 \mu \mathrm{m}$ ) and incubated overnight on supplemented BHI agar. Mating filters were resuspended in broth and plated on selective media containing $10 \mu \mathrm{g} / \mathrm{ml}$ of chloramphenicol, or $5.0 \mu \mathrm{g} / \mathrm{ml}$ of $\mathrm{AM}$, or combinations of AM and CM, with $100 \mu \mathrm{g} / \mathrm{ml}$ of streptomycin. The recipient haemophilus strain was a streptomycin-resistant (MIC $100 \mu \mathrm{g} / \mathrm{ml})$ and ampicillin $(\mathrm{MIC}=0.01$ $\mu \mathrm{g} / \mathrm{ml}$ )/chloramphenicol (MIC $=4 \mu \mathrm{g} / \mathrm{ml}$ )-susceptible Rd haemophilus isolate, strain MAP (provided by Dr. Arnold Smith Seattle, WA). No specific selective plates for tetracycline were utilized. Background rates of mutation to streptomycin resistance among donor strains and mutation to $\mathrm{CM}$ and $\mathrm{AM}$ resistance among recipient strains were calculated by plating $10^{8}$ cell to each of 10 plates containing single antibiotics in the concentrations listed above. Spontaneous mutation frequencies for streptomycin among Rd cells was $10^{-9}$, while no mutants resistant to AM or CM were recognized. Transformation was performed according to the method of Herriot et al. (15) utilizing the same strains. Transformation experiments were attempted twice for each autopsy strain (i.e. nonplasmid-bearing isolates, resistant to $\mathrm{AM}$ and $\mathrm{CM}$ ). Approximately $1.0 \mathrm{mg}$ of crude lysate chromosomal DNA was utilized for each experiment. Transformation of the recipient Rd MAP strain with a chromosomal mediated rifampin resistant marker from a previously transformed strain of haemophilus Eagan (Ela) occurred at an average rate of 1.2 transformants per $10^{8}$ recipient cell.

\section{RESULTS}

Table 1 lists the haemophilus isolates from the infant, including the date, anatomic site, antibiotic susceptibility, CAT and $\mathrm{BL}$ production, and the presence or absence of plasmid DNA. All strains were identical for both major and minor protein bands by OMP SDS-PAGE analysis and were subtype 5L. All nine isolates were susceptible to cefotaxime, ceftizoxime and ceftriaxone (MIC $0.05 \mu \mathrm{g} / \mathrm{ml}$ ) and rifampin (MIC $0.05 \mu \mathrm{g} / \mathrm{ml}$ ). Strains isolated from the blood and synovial fluid during the first hospitalization were susceptible to AM, CM, tetracycline, and TMP/ SMX. None of these strains contained plasmid DNA and neither CAT or BL was detected in any isolate. In contrast, strains isolated during the infant's second admission (i.e. meningitis) all produced CAT and BL. All organisms during this hospitalization were highly resistant to AM and CM and had plasmid DNA detected. In addition, these strains were resistant or had intermediate activity against tetracycline, SMX, and TMP/SMX by disk susceptibility testing; all isolates were susceptible to TMP alone. In contrast, although the two strains isolated from the middle ear at the time of autopsy also produced CAT and BL, neither organism had detectable plasmid DNA.

Figure 1 displays the electrophoresis of DNA from all sample isolates and two transconjugants. All identified plasmids were 
Table 1. Chronologic collection, anatomical site or source, and antibiotic susceptibility for 10 serial isolates of Hib, OMP, subtype $5 L$ from a 4-month-old infant

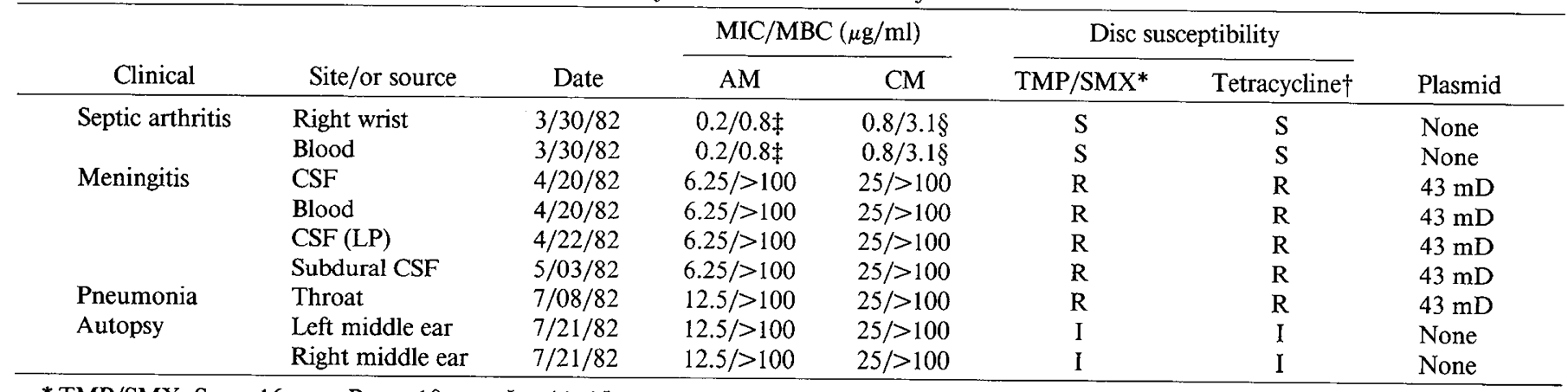

* TMP/SMX, $\mathrm{S}=\geq 16 \mathrm{~mm} ; \mathrm{R}=\leq 10 \mathrm{~mm} ; \mathrm{I}=11-15 \mathrm{~mm}$.

$\dagger$ Tetracycline, $S=\geq 19 \mathrm{~mm} ; \mathrm{R}=\leq 14 \mathrm{~mm} ; \mathrm{I}=15-18 \mathrm{~mm}$.

$\$$ BL negative.

$\S$ CAT negative.

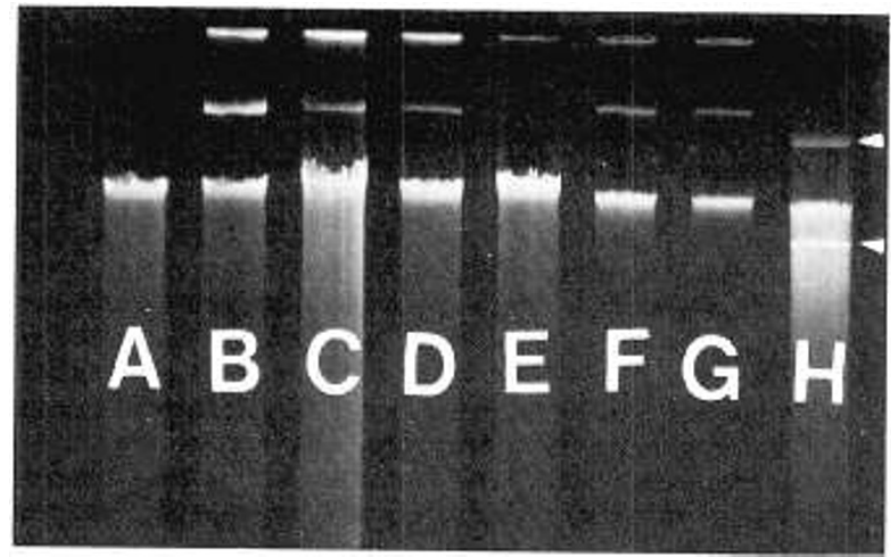

Fig. 1. Lane $A$ to $H$ from left to right. Lane $A$ AM-CM susceptibile isolate from right wrist; lanes $B, C, D$, AM-CM-tetracycline-TMP/SMX resistant isolates from blood CSF and subdural effusion, respectively; Lane $E$, AM-CM-resistant isolate from autopsy specimens; lanes $F$ and $G$, AM-CM-resistant Rd haemophilus transconjugants crossed with AMCM-resistant isolates from lane $B$; and lane $H, E$. coli plasmid standards with arrows indicating reference plasmids of 34 and $7.5 \mathrm{mD}$ in size (from top to bottom).

approximately $42-44 \mathrm{mD}$ in size. Plasmid-bearing strains, isolated during the second hospitalization transferred plasmid DNA in conjugation experiments at an average frequency of 2.4 transconjugants per $10^{5}$ recipient cells. AM and CM resistance were cotransferred in all transconjugates from 102 colonies screened from AM-streptomycin plates and 89 colonies screened from CM-streptomycin plates. Tetracycline resistance was detected in $152(80 \%)$ of these 191 transconjugates. TMP/SMX resistance or intermediate activity was found in 61 of 191 transconjugants $(32 \%)$ utilizing disc susceptibility criteria listed in Table 1. AM/CM-resistant strains (CAT and BL positive) isolated from the middle ear at autopsy did not transfer either resistance determinant by conjugation in four crosses. However, transformation of haemophilus Rd cells with DNA from autopsy isolates transferred resistance from plasmid-negative cells at a very low frequency. Five colonies of AM-CM-resistant Rd haemophilus cells were isolated in four experiments (frequency = 1.2 transformed cell $/ 10$; 8 recipients); none of these five transformants was resistant to TMP, TMP/SMX, or SMX and no AM-CM-resistant transformant had extrachromosomal DNA.

\section{DISCUSSION}

Plasmid encoded resistance has been demonstrated for most AM-CM-resistant strains of haemophilus organisms (4-6). AM resistance is most frequently due to plasmid encoded production of TEM-1 type BL on large conjugative plasmids. Similarly, CM resistance also is frequently due to plasmid encoded production of CAT. The molecular weight of the plasmid identified in our strains, and the multiple resistance pattern is similar to another strain described from a California child (3). Although the incidence of combined $\mathrm{AM}$ and $\mathrm{CM}$ resistance in $H$. influenzae remains low in the United States, studies in Spain have revealed frequencies as high as 40 to $50 \%(16,17)$. In Spain, AM and CM coresistance frequently occurs concomitantly. Thus although AM resistance may occur alone in individual isolates, resistance to CM alone is rare (17). In addition, Spanish haemophilus isolates frequently are coresistant to tetracycline, sulfonamides, and the combination of TMP/SMX, in addition to AM and CM. Tetracycline resistance among haemophilus strains has been shown to be usually related to the class-b type determinant which is common among enteric isolates (18). Coresistance to TMP/ SMX and tetracycline has been observed in greater than $50 \%$ of isolates in Spanish studies $(15,16)$ but whether this is due to TMP, sulfonamide resistance, or both, has not been clarified.

Stuhy (19) has demonstrated the frequent incorporation of AM, CM, and tetracycline resistance determinants encoded by plasmids into the bacterial chromosome of $H$. influenzae and Haemophilus parainfluenzae (19). The evidence suggesting incorporation of the plasmid DNA sequences encoding AM (BL) and $\mathrm{CM}$ (acetyltransferase) resistance into the chromosomal genome in our strains is based on three observations. First, plasmids capable of transferring these resistance markers were observed only in clinical isolates, whereas plasmid copies were not observed in isolates from the autopsy strains. Second, resistance to AM and CM were transferred at relatively high frequencies by conjugation (i.e. cell-to-cell contact), and transconjugants bore a plasmid identical to donor strains and expressed resistance to these antibiotics. In contrast, resistance markers could not be transferred by conjugation from any autopsy isolates in which plasmids were not visualized. Third, low level transfer of AM and $\mathrm{CM}$ resistance using the autopsy (plasmid negative) strains was accomplished only by transformation. Characteristically chromosomal markers are transferred by transformation whereas large plasmids are transmitted by conjugative mechanisms. Although a possible transfer of chromosomal genomes by a conjugative, cell-to-cell mechanism has been described in a limited number of strains (20), it is uncertain whether such a conjugative mechanism frequently occurs in nature. We did not perform experiments to identify homologous DNA sequences between plasmid and chromosomal DNA by hybridization. However, Mendelman et al. (3) have demonstrated incorporation of a 43 Mdal plasmid encoding multiple resistance into transconjugants of a recombinant proficient recipient strain and as noted previously, Stuhy (19) has shown that plasmids can be incorporated into haemophilus chromosomes. 
In our patient, it is probable that his initial infection was caused by an unrecognized mixed population of resistant and susceptible haemophilus cells. Presumably, antibiotic therapy led to the selection of an antibiotic-resistant population with subsequently relapsing episodes of infection. Alternatively, a resistance transposon may have been acquired by colonizing haemophilus pathogens from respiratory or enteric flora. Results of sulfonamide and TMP susceptibility testing are difficult to interpret in haemophilus species. However, the failure of clinical response during TMP/SMX therapy, coupled with the apparent resistance or intermediate susceptibilities of the recovered isolates and some transconjugants to TMP/SMX, suggests that the latter organisms were most likely resistant to this drug. Resistance to SMX or TMP alone may have been sufficient for the infection to fail to respond to the combination and may explain the variable susceptibility results. Resistance to AM and CM (and presumptively tetracycline and TMP/SMX) were encoded on a plasmid of approximately $42-44 \mathrm{mD}$. AM and CM were cotransferred in all conjugations experiments but resistance to tetracycline or TMP/SMX varied from 80 to $30 \%$, respectively.

$\mathrm{AM}$ and $\mathrm{CM}$ were the only resistance determinants apparently incorporated into the chromosome. Strains isolated during all episodes of infection had the same outer membrane protein subtype $5 \mathrm{~L}$ and had identical minor proteins. Therefore, they were presumptively the same causative strain throughout all episodes. The ability to transform AM and CM susceptible $\mathrm{Rd}$ cells by whole cell (chromosomal) DNA from AM-CM resistant (plasmid negative) strains suggest that there was incorporation of the plasmid determinant into the chromosome of isolates from the middle ear.

This case underscores the need for repeated clinical and cultural evaluation of children receiving therapy for bacterial meningitis with conventional antibiotic regimens. AM alone was employed as the initial antibiotic to treat this infant's septic arthritis. Although the continued use of AM was justified by initial tests suggesting susceptibility to this antibiotic, the combination of AM and CM (21), or a third generation cephalosporin (22), are recommended for initial use. TMP/SMX therapy of haemophilus infections has been noted to be associated with a higher incidence of therapeutic failure than conventional regimens. This may be due either to resistance to TMP, SMX, or both agents (23). The clinical course and results of follow-up lumbar punctures in this child suggested an initial clinical response to TMP/SMX. The subsequent recognition of the clinical and bacteriologic failure of TMP/SMX therapy was delayed and was apparent only with repeated examinations of the spinal fluid. The potential for integration of resistance determinants to AM and $\mathrm{CM}$ into the haemophilus chromosome is a cause for clinical concern. Such resistance theoretically may be more stable and transferred successfully to daughter cells with each chromosomal division. Such a phenomenon may contribute to an increasing incidence of multiply-resistant strains in areas where AM and $\mathrm{CM}$ are used extensively.

\section{REFERENCES}

1. Overturf GD, Wilkins J, Leedom JM, Ivler D, Mathies AW 1975 Susceptibility of Hemophilus influenzae type b to ampicillin at Los Angeles CountyUniversity of Southern California Medical Center. J Pediatr 87:297-300

2. Tomeh OM, Starr SE, McGowan JE. Terry PM. Nabmias AJ 1974 Ampicillinresistant Hemophilus influenzae type b infection. JAMA 229:195-300

3. Mendelman PM, Doroshow CA, Gandy SL, Syriopoulou V, Weigen CP. Smith AL 1984 Plasmid-mediated resistance in a multiply resistant Haemophilus influenzae causing meningitis: molecular characterization of one strain and review of the liturature. J Infect Dis 150:30-39

4. Schiefile GN, Fusseal SJ, Roberts MC 1982 Characterization of ampicillinresistant Haemophilus parainfluenzae. Antimicrob Agents Chemother 21:734-739

5. Elwell LP, DeGraff J, Seibert D and Falkow S 1975 Plasmid-linked ampicillin resistance in Haemophilus influenzae type b. Infect Immun 12:404-410

6. Roberts MC, Swenson CD, Owens LM, Smith AL 1980 Characterization of chloramphenicol-resistant Haemophilus influenzae. Antimicrob Agents Chemother 18:610-615

7. Jones RN (Chairman, NCCLS Subcommittee on Disk Diffusion Susceptibility Testing) 1984 Performance Standards for Antimicrobial Disk Susceptibility Tests, 3rd ed. National Committee for Clinical Laboratory Standards, Washington, D.C., 4:369-406

8. Rocco V, Overturf GD 1983 Chloramphenicol antagonism of the bactericidal activity of ampicillin against $H$. influenzac. Antimicrob Agents Chemother 21:349-351

9. Department of Chemotherapy, Hoffman-LaRoche, Inc 1982 A Revicw of the Bactericidal Nature of Bactrim. Hoffman La-Roche Inc, Nutley, NJ, pp 1421

10. Loeb M, Zachary A, Smith D 1981 Isolation and partial characterization of outer and inner membranes from encapsulated Haemophilus influenzae type b. J Bacteriol 145:596-604

11. Barenkamp SJ, Munson RS Jr. Granoff DM 1981 Subtyping isolates of Haemophilus influenzae type b by outermembrane protein profiles. J Infect Dis 143:668-676

12. Thornberry C, Kirven LA 1974 Ampicillin resistance in Haemophilus influenzae as determined by a rapid test for beta-lactamase production. Antimicrob Agents Chemother 6:653-654

13. Burns JL, Rubens CE. Mendelman PM. Smith AL 1986 Cloning and expression in $E$. coli of a gene encoding nonenzymatic chloramphenicol resistance from $P$. aeruginosa. Antimicrob Agents Chemother 39:445-450

14. Meyers JA, Sanchez D, Elwell LP, Falkow S 1976 Simple agarose gel electrophoretic method for the identification and characterization of plasmid deoxyribonucleic acid. J Bacteriol 127:1529-1537

15. Herriot RM, Meyer EM, Vogt M 1984 Defined nongrowth media for stage II development of competence in Haemophilus influenzae. J Bacteriol 101:517-524

16. Campos J, Garcia-Tornel D, Semfeliu I 1984 Susceptibility studies of multiply resistant Haemophilus influenzae isolated from pediatric patients and contacts. Antimicrob Agents Chemother 25:706-709

17. Campos J, Garcia-Tornel D, Gairi JM. Fabregues I 1986 Multiply resistant Haemophilus influenzae type $\mathrm{b}$ causing meningitis: comparative clinical and laboratory study. J Pediatr 108:897-902

18. Marshall B, Roberts M, Smith A, Levy SB 1984 Homegeneity of tetracyclineresistance determinants in Haemophilus species. J Infect Dis 149:1028-1029

19. Stuhy J 1980 Chromosomally integrated plasmids are common in antibioticresistant Haemophilus influenzae. J Bacteriol 142:925-930

20. Albritton WL, Setlow JK, Slancy L 1982 Transfer of Haemophilus influenzae chromosomal genes by cell-to-cell contact. J Bacteriol 152:1066-1070

21. Committee on Infectious Disease 1976 Current status of ampicillin-resistant Hemophilus influenzae type B. Pediatrics 57:417

22. Overturf GD 1986 Newer cephalosporins in neonatal and pediatric meningitis. Infect Dis Newsletter 5:17-20

23. Levitz RE, Quintiliani R 1984 Trimethoprim-sulfamethoxazole for bacterial meningitis. Ann Intern Med 100:881-890 